\title{
Study on Heat Transfer Performance of Orifice Plate Configuration in Plenum Chamber
}

\author{
Hong Zhang ${ }^{1, a}$, Jianwei Shi ${ }^{1, b,}$, , Chenyu Wang ${ }^{1, c}$, Junming Zhang ${ }^{1, d}$ \\ 1Department of Mechanical Engineering, Dalian University of Technology, Dalian 116024, China \\ ajxzh@dlut.edu.cn, bssjjww112266@126.com, ‘wcyduanmuqi@163.com, dzjmsci@sina.com \\ ${ }^{*}$ Corresponding author
}

\begin{abstract}
Keywords: Lithium battery film, Plenum chamber, Convective heat transfer, Orifice plate configuration.
\end{abstract}

\begin{abstract}
The plenum chamber, as an important part in the production process of lithium battery films, directly impacts the efficiency of hot air circulation and the temperature field uniformity in the thermal insulating room. Studies confirm the orifice plate configuration of the plenum chamber affects the heat transfer performance, but the influence rules of parameters (e.g. staggered angle, opening ratio) on the heat transfer performance are unknown yet. In this regard, an in-depth study was conducted to explore the effects of orifice plate configuration on the heat transfer performance of films. Firstly, a plenum chamber model in the thermal insulating room was established based on the actual production line of lithium battery films. Then numerical simulation was studied via the computational fluid dynamics method. The numerical simulation method and the modeling accuracy were verified by comparing the simulated and experimental data of orifice jet velocity. Finally, based on the numerical model, how the regular changes of staggered angle and spanwise row number would affect the intensity, uniformity and efficiency of film heat transfer were systematically revealed. This study provides reference for structural optimization of the orifice plate configuration.
\end{abstract}

\section{Introduction}

In the wet-laid production line of lithium battery film, extrusion die, plenum chamber groups in the insulating room, chain movement control, etc. are the key to the overall film technology [1]. Among them, the main functions of the plenum chamber in the insulating room are to convert the dynamic pressure of the airflow in the fan duct into the static pressure as much as possible, stabilize and evenly distribute the airflow, and it is a key production equipment for ensuring even temperature at the outlet of the hot-air heating system, as well as has a direct impact on the stretching stability of the film forming process [2].

At present, the plenum chamber in the production line of lithium battery film mostly relies on orifice plate to realize the forced convective heat transfer on film, however, the selection of structural parameters of the orifice plate is usually given according to experience, which needs to be adjusted and improved repeatedly. Extensive literature presents the heat transfer performance of orifice plate through experimental as well as numerical studies. Shao Jidong et al. estimated the velocity distribution uniformity of orifice plate jet and recommended the optimal parameters of orifice plate are $d=12 \mathrm{~mm}, l=26 \mathrm{~mm}, t=6 \mathrm{~mm}$, but if the thickness of the orifice plate is $6 \mathrm{~mm}$ (that is, $t=6 \mathrm{~mm}$ ), it will make the plenum chamber too heavy, as a result, it is unfavourable to be processed, installed and fixed [2]. [3-5] presented a comprehensive investigation on the effect of ratio of plate thickness to diameter, diameter of perforated holes and height of plenum chamber on jet impingement heat transfer performance, however, the field was confined to the inner plenum chamber and could not figure out the characteristics of the flow field outside the chamber. [6,7] considered the effect of jet-to-target distance, distance between orifices both in flow direction and spanwise direction, etc. on the heat transfer performance, but the distance of orifice to target surface is small and the target surface is mostly made of metal material, which is not suitable for the plenum chamber in lithium battery film production line where Reynolds number is larger and thermal conductivity of film is lower. Furthermore, Goodro et al. [8,9] studied the effect of Reynolds number, Mach number, hole spacing 
and temperature ratio on jet impingement heat transfer performance during 2008-2015. Lee et al. [10] considered the effects of crossflow on heat transfer distributions, experimental results showed that local, line-averaged, and spatially averaged Nusselt numbers have complex dependence on hole spacing, jet-to-target distance and impingement jet Reynolds number. Previous studies about orifice plate configurations didn't reveal the effects of parameters such as staggered angle and opening ratio on heat transfer of the target surface.

In the light of the very complicated airflow distribution in the insulating room under the jet impingement of orifice plate, it's difficult to measure and observe the internal flow field and the boundary layer in the stagnant area of film. In view of this, this paper studies the orifice plate configurations with staggered angle and number of spanwise rows varying regularly based on CFD (Computational Fluid Dynamics) method. The simulation results of orifice jet velocity and experimental results are compared to verify the effectiveness of numerical simulation methods. On this basis, the airflow distribution in the three-dimensional flow field and its effect on the heat transfer of the film were investigated by comparing the surface heat transfer coefficient of the film with different orifice plate configurations.

\section{Modeling and numerical calculation method}

\subsection{Modeling of plenum chamber in insulating room}

Figure 1 shows the appearance and partial break-out section view of the insulating room in the film production line, in which the plenum chambers were arranged symmetrically. Hot air provided by the fans set besides the insulating room blew into the chamber and formed air jet impingement acting on film after rectification through the orifice plate, as shown in Figure 2, where the direction of the arrow indicates the moving direction of the film.

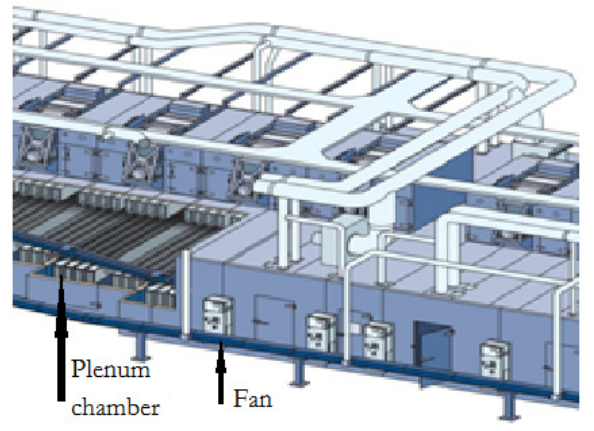

Fig. 1. Insulating room in the film production line

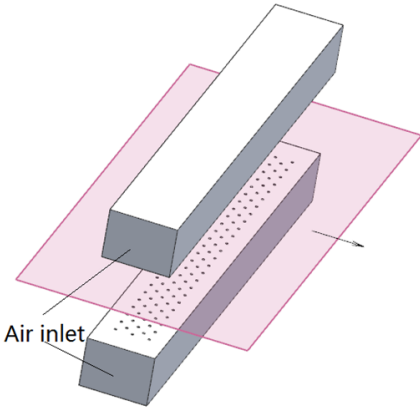

Fig. 2. Schematic diagram of plenum chamber

In order to study the effects of different orifice plate configurations on the heat transfer of film easily and efficiently, the local features of the plenum chamber and the insulating room are simplified. Since the film thickness ranges from 7 to $25 \mu \mathrm{m}$ and the extrusion die opening is only $0.6 \mathrm{~mm}$ [11] when working, and the upper and lower symmetrical arrangement of the plenum chamber has the same situation during the convective heat transfer on film, we can select the case of single-sided heat transfer on film to study the effects of orifice plate. The model of plenum chamber in insulating room according to the existing lithium battery film production line project is established as shown in Figure 3 , with the size parameters as follows: inlet size of plenum chamber is $400 \times 300 \mathrm{~mm}$; the front and rear walls of chamber fit the insulating room well and the length is $2780 \mathrm{~mm}$; the size of the insulated room is $800 \times 2780 \times 607.5 \mathrm{~mm}$, in which the distance from the horizontal plane of the orifice plate to the film is $257.5 \mathrm{~mm}$.

According to the actual orifice plate configuration used in the project, the orifice plate shown in Figure 4 is designed to study the effects of staggered angle and number of spanwise rows on heat transfer of the film. Where $S_{1}$ is the flow spacing, $S_{2}$ is the spanwise spacing, and $\alpha$ is the staggered angle. The thickness of the orifice plate is $1.2 \mathrm{~mm}$, the diameter of the orifice is $15 \mathrm{~mm}$, and the structural parameters of each orifice plate configuration are shown in Table 1. 


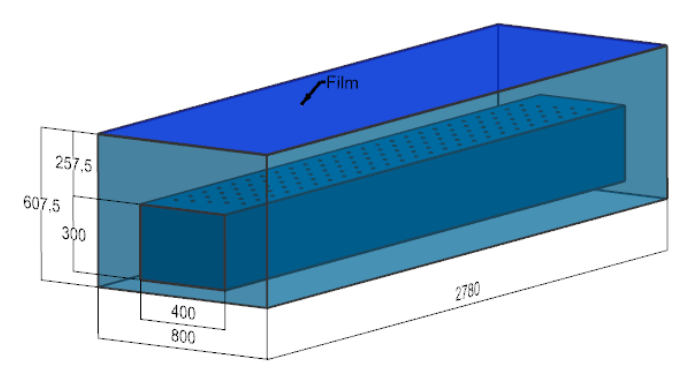

Fig. 3. Model of plenum chamber in Insulating room

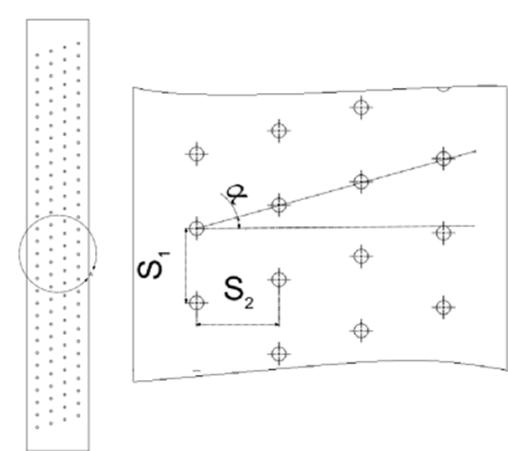

Fig. 4. Diagram of orifice plate parameters

Table 1. Parameters of orifice plate

\begin{tabular}{ccccccccccccc}
\hline Type & $\mathrm{A}$ & $\mathrm{B}$ & $\mathrm{C}$ & $\mathrm{D}$ & $\mathrm{E}$ & $\mathrm{F}$ & $\mathrm{G}$ & $\mathrm{H}$ & $\mathrm{I}$ & $\mathrm{J}$ & $\mathrm{K}$ & $\mathrm{L}$ \\
\hline$S_{1}(\mathrm{~mm})$ & 120 & 120 & 120 & 120 & 100 & 100 & 100 & 100 & 80 & 80 & 80 & 80 \\
\hline$S_{2}(\mathrm{~mm})$ & 102 & 102 & 102 & 102 & 85 & 85 & 85 & 85 & 68 & 68 & 68 & 68 \\
\hline$\alpha\left({ }^{\circ}\right)$ & 0 & 15 & 30 & 45 & 0 & 15 & 30 & 45 & 0 & 15 & 30 & 45 \\
\hline Number of spanwise rows & 3 & 3 & 3 & 3 & 4 & 4 & 4 & 4 & 5 & 5 & 5 & 5 \\
\hline Number of orifices & 63 & 63 & 63 & 63 & 100 & 100 & 100 & 100 & 155 & 155 & 155 & 155 \\
\hline
\end{tabular}

\subsection{Numerical calculation method}

The insulating room includes the pre-heating zone, the stretching zone and the heat-setting zone. The largest airflow velocity in the insulating room locates in the core section of the orifice jet in the preheating zone, reaching $80 \mathrm{~m} / \mathrm{s}$ at the maximum blowing air volume of the fan and the corresponding Mach number is 0.24; therefore, the hot air can be regarded as incompressible fluid. In this paper, the software Fluent 18.0 is used to solve the above three-dimensional incompressible Reynolds averaged Navier-Stokes equations. The common equations of the continuous equations, momentum equations and energy equations [12] are written as:

$$
\frac{\partial \rho \bar{\phi}}{\partial t}+\frac{\partial\left(\rho \overline{u_{j}} \bar{\phi}\right)}{\partial x_{j}}=\frac{\partial}{\partial x_{j}}\left(\Gamma \frac{\partial \rho \bar{\phi}}{\partial x_{j}}-\rho \overline{u_{j}^{\prime}} \overline{\phi^{\prime}}\right)+S .
$$

Where, superscript "-"means the time mean value, and "' "means the ripple quantity. The instantaneous value $\phi$, the mean value ${ }^{-} \phi$ and the ripple quantity $\phi$ ' satisfy the relationship: $\phi$ $=\bar{\phi}+\phi$.

The supplying air volume of plenum chamber in different zones of the insulating room is controlled by centrifugal fans and variable frequency motors. In this paper, the preheating zone is used to study the convective heat transfer on the film. The medium is $150^{\circ} \mathrm{C}$ hot air, the density $\rho=0.8347 \mathrm{~kg} / \mathrm{m}^{3}$, the dynamic viscosity $\mu=2.4 \times 10^{-5} \mathrm{~Pa} \cdot \mathrm{s}$, and the orifice diameter $D=15 \mathrm{~mm}$. According to

$$
\operatorname{Re}=\frac{\rho u D}{\mu},
$$

The calculated inlet Reynolds number of the plenum chamber exceeds 60000 . Hot air in insulating room develops fully after heating the film and then flows into the hot air circulation system, so the k$\varepsilon$ standard turbulence model is selected. The film wall is analyzed using the method of standard wall function. The solution process is steady-state and the hot air in the computational domain is considered as incompressible fluid of low velocity, so the Pressure-Based type and SIMPLE algorithm are adopted.

The overall mesh of the global model is shown in Figure 5. In order to achieve the ideal calculation accuracy and make rational use of computing resources, the local mesh density on the 
orifice plate is increased, as shown in Figure 6. The orifices of the plenum chamber and the heat transfer surface at the border of film and flow field are regarded as coupled surfaces.

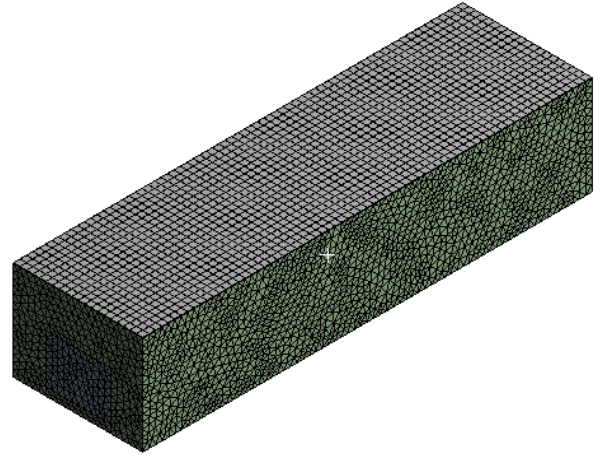

Fig. 5. Overall mesh of the global model

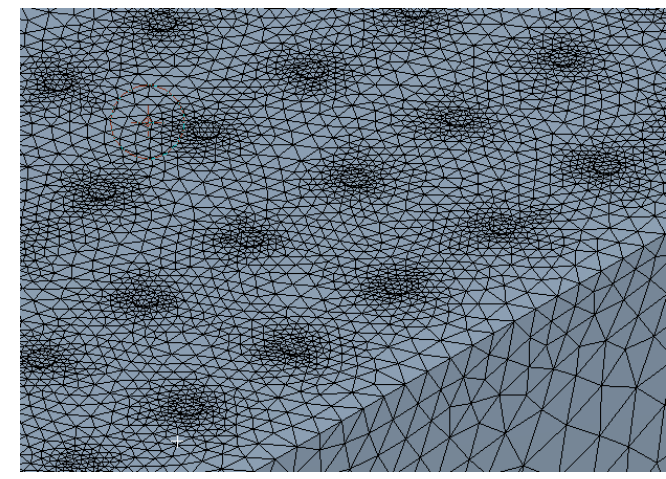

Fig. 6. Local mesh density on the orifice plate

The velocity inlet is adopted in this model according to the actual flow situations of supplying air and return air, and the medium temperature of supplying hot air is set as $423 \mathrm{~K}$. The walls around the insulating room are regarded as outlets and are set as outflow type due to the unknown flow velocity and pressure on the boundaries. In this paper, only the case of single-sided heat transfer of the film is considered, so the interface of the film and flow field is set as coupled surface, and the thermal boundary conditions on the other surfaces of the film are set as convective heat transfer conditions. Here the free flow temperature is set as the ambient temperature $300 \mathrm{~K}$ and the heat transfer coefficient is subject to the natural convection heat transfer with the atmosphere, which is set as $10 \mathrm{~W} / \mathrm{m}^{2} \cdot \mathrm{K}$ [13].

\subsection{Model correctness verification}

The orifice plate of plenum chamber in the production line of lithium battery film is shown in Figure 7. The chamber is equipped with an air uniform plate (as shown by the dotted line in Figure 7) and has bilateral air supply by the fan. To verify the correctness of the simulation method, the model of the plenum chamber with this kind of orifice plate configuration was simulated and analyzed, and the orifice jet velocity was compared with the results of experiments. Of all the comparisons, the measuring points numbered 1-25 are located at the orifices of the rows in flow direction numbered 1 , $8,16,24$ and 31 respectively. The total pressure of the centrifugal fan is $1300 \mathrm{~Pa}$, the max airflow rate is $15000 \mathrm{~m}^{3} / \mathrm{h}$, and the speed of back bending blade is $1450 \mathrm{rpm}$, supplying air simultaneously to the upper and lower plenum chamber symmetrically arranged. The boundary conditions are given based on function efficiency $85 \%$, taking into account that the efficiency of this type of fan is usually $81 \%-88 \%$. Figure 8 and Figure 9 show the comparison of the simulated and experimental results when the motor frequency is $30 \mathrm{~Hz}$ and $50 \mathrm{~Hz}$ respectively, in which the relative errors between the simulated and experimental results are both less than 3.8\%. Considering the actual fan efficiency, numerical calculation error and the inevitable error in the experimental measurement, the model can reflect the actual flow field conditions in the insulating room.

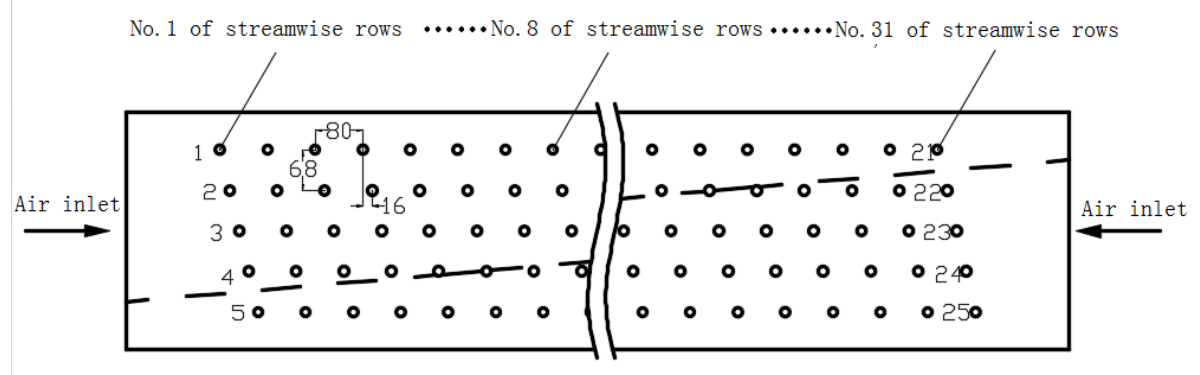

Fig. 7. Orifice plate configuration of plenum chamber in the film production line 


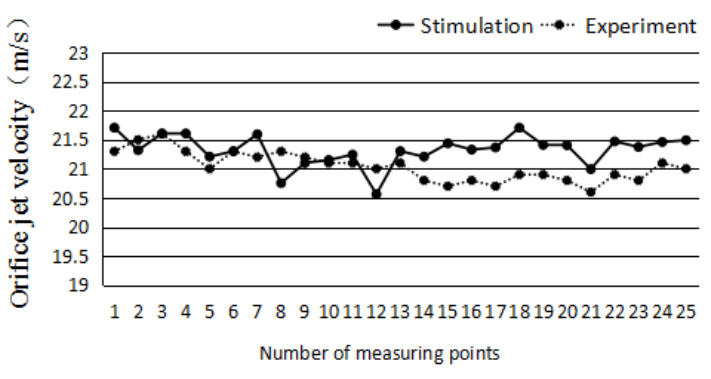

Fig. 8. Comparison chart when motor frequency is $30 \mathrm{HZ}$

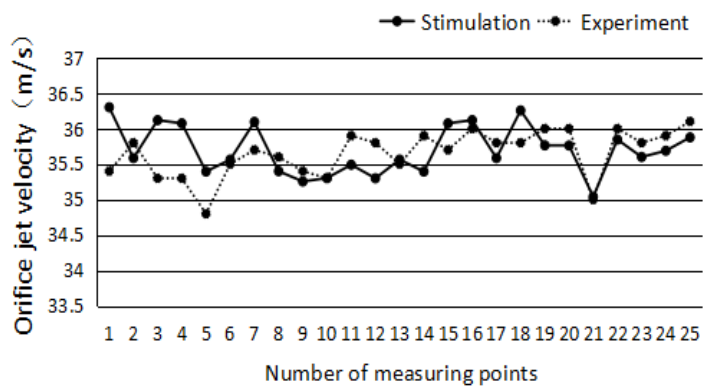

Fig. 9. Comparison chart when motor frequency is $50 \mathrm{HZ}$

\section{Numerical simulation results and analysis}

\subsection{Convective heat transfer performance}

According to the results of flow field simulation, the average Nusselt number $\bar{N} u$ of the convective heat transfer between the film and the hot air in the insulating room under different boundary conditions can be obtained. The Nusselt number, calculated by the equation below,

$$
N u=\frac{h L}{k}=\frac{\left.\frac{\partial\left(T_{s}-T\right)}{\partial y}\right|_{y=0}}{\frac{T_{s}-T_{\infty}}{L}},
$$

is the ratio of conductive heat transfer resistance and convective heat transfer resistance on the boundary surface during heat transfer, which characterizes the intensity of convective heat transfer. Where, $h$ is the surface convective heat transfer coefficient $\left(\mathrm{W} / \mathrm{m}^{2} \bullet \mathrm{K}\right), L$ is the characteristic length $(\mathrm{m}), k$ is the thermal conductivity of the medium $(\mathrm{W} / \mathrm{m} \bullet \mathrm{K})$, and the physical meaning of the remaining parameters is shown in Figure 10. In this simulation, the free stream temperature $T_{\infty}$ is the hot air temperature $423 \mathrm{~K}$, and $T_{s}$ is the initial temperature of the film which is patched as $403 \mathrm{~K}$. Considering the different characteristic lengths of the thermal boundary layer on the film when the orifice plate configurations and boundary conditions are different, the average $h$ of film (hereinafter referred to as $h$ ) is used to evaluate the convection heat transfer intensity of different orifice plate configurations.

Under the same inlet velocity, the Reynolds number of the initial crossflow section of the plenum chamber is the same, and $h$ corresponding to each orifice plate is shown in Figure 11. The number of opening orifices in each orifice plate is different, so that the average jet Reynolds number $\operatorname{Re}_{D}$ shown in Table 1 numbered A-L is different. The average jet Reynolds number is defined as

$$
R e_{D}=\frac{4 m}{n \pi D \mu} .
$$

Where, $D$ is the diameter of the orifice $(\mathrm{m}), n$ is the number of orifices, $\mu$ is the dynamic viscosity of hot air $(\mathrm{Pa} \bullet \mathrm{s})$ and $m$ is the mass flow rate at the orifice plate $\left(\mathrm{kg} / \mathrm{m}^{2} \bullet \mathrm{s}\right)$. It can be seen from the figure that $h$ and the average jet Reynolds number of the orifice plate have the same trend under different configurations: under the same inlet velocity, the jet Reynolds number decreases with the increase of the number of orifices and $h$ decreases; as the inlet velocity increases, the mass flow rate at the orifice plate $m$ increases, while the jet Reynolds number increases, so does $h$. The average $h$ of film first increases and then decreases with the increase of the staggered angle when the number of spanwise rows is 3 or 4 , and reaches the maximum at $30^{\circ}$. When the number of spanwise rows is $5, h$ is less affected by the staggered angle. 


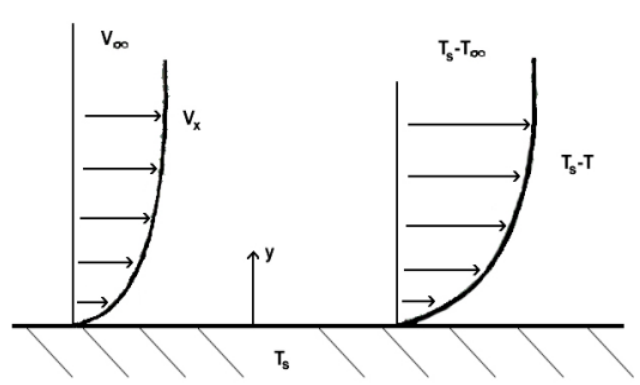

Fig. 10. Thermal boundary layer

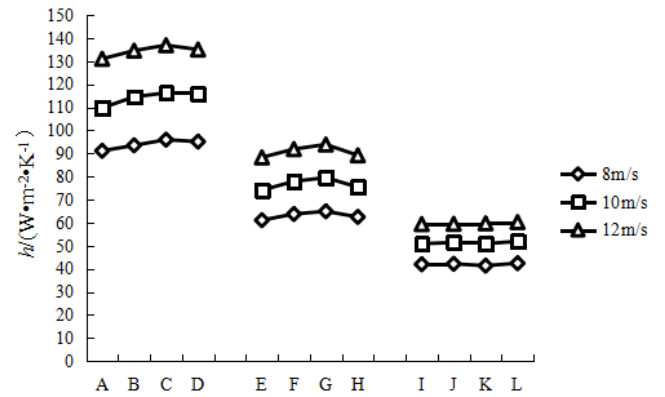

Fig. 11. $h$ under the same inlet velocity

Under the same jet Reynolds number, $h$ corresponding to each orifice plate is shown in Figure 12. Like the same velocity inlet conditions, $h$ increases first and then decreases with the increase of staggered angle, and reaches the maximum at $30^{\circ}$. When the number of spanwise rows is $5, h$ varies inconspicuously with the increasing staggered angle. As shown in Figure 13, $h$ for the number of spanwise rows 4 is higher than that for the number of spanwise rows 3 and 5 when the staggered angles are $0^{\circ}, 15^{\circ}, 30^{\circ}, 45^{\circ}$ respectively. It shows that the heat transfer effect is better when the number of spanwise rows is 4 under the same jet Reynolds number.

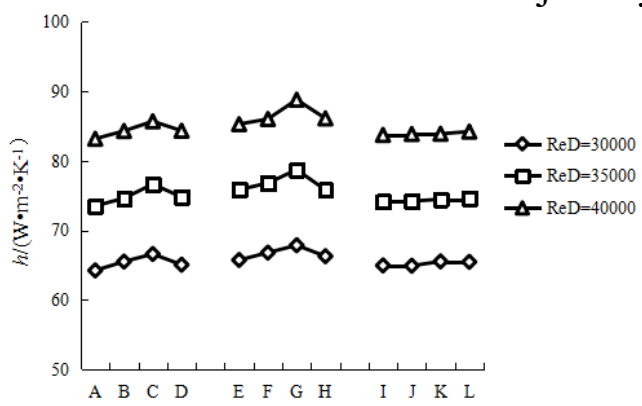

Fig. 12. $h$ under the same number of spanwise rows

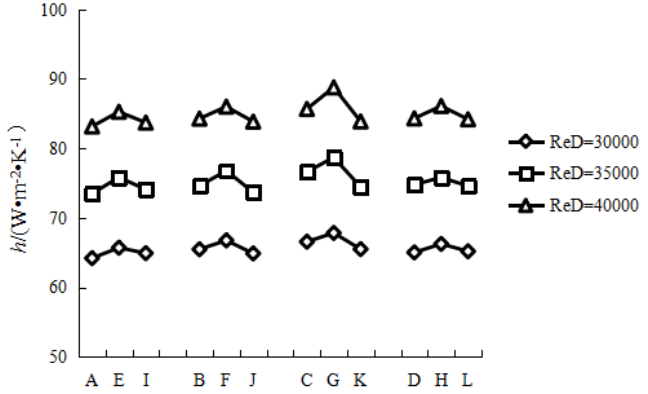

Fig. 13. $h$ under the same staggered angle

\subsection{Heat transfer uniformity}

To quantify the heat transfer uniformity of each configuration, the degree of dispersion of $h$ is defined as follows:

$$
C_{v}=\frac{\sigma}{\mu} \text {. }
$$

Where, $\sigma$ is the standard deviation of $h$; $\mu$ is the average value of $h$; the smaller value of $C_{\nu}$ corresponds to the better uniformity. Figure 14 and Figure 15 show the dispersion degree $C_{v}$ of $h$ under the same inlet velocity and jet Reynolds number, respectively. It can be seen from the figure that under the same boundary conditions, the minimum dispersion degree $C_{v}$ occurred at the staggered angle of $45^{\circ}$, $30^{\circ}$ and $30^{\circ}$ when the corresponding numbers of spanwise rows are 3, 4, and 5 respectively. When the number of spanwise rows is $3, C_{v}$ is getting smaller with the increase of the staggered angle, followed by the increasingly better uniformity of heat transfer. When the number of spanwise rows is 4 or $5, C_{v}$ decreases when the staggered angle increases from $0^{\circ}$ to $30^{\circ}$, while increases when the staggered angle increases from $30^{\circ}$ to $45^{\circ}$. It is thus clear that when the opening ratio is low, like the configurations when the number of spanwise rows is 3 herein, the increase of the staggered angle can reduce the blind coverage of the hot air on the film and improve the heat transfer uniformity with a certain number of orifices. In the configurations when the number of spanwise rows is 4 or 5 , which have a larger number of orifices, increasing the staggered angle can improve the heat transfer uniformity to some extent, but in the case studied in this paper, increasing the staggered angle over $30^{\circ}$ will reduce the heat transfer uniformity. 


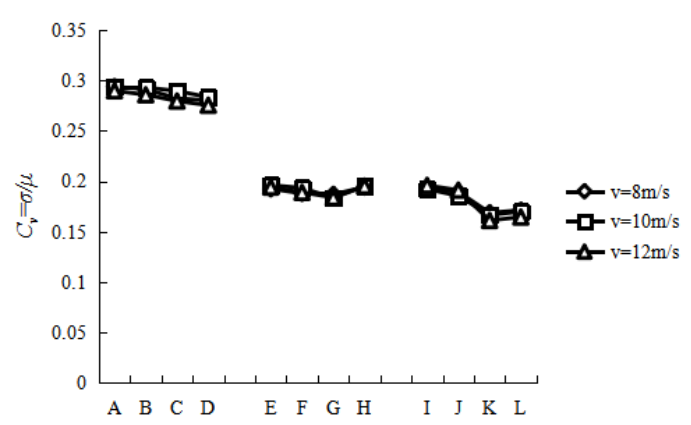

Fig. 14. Dispersion of $h$ under the same inlet velocity

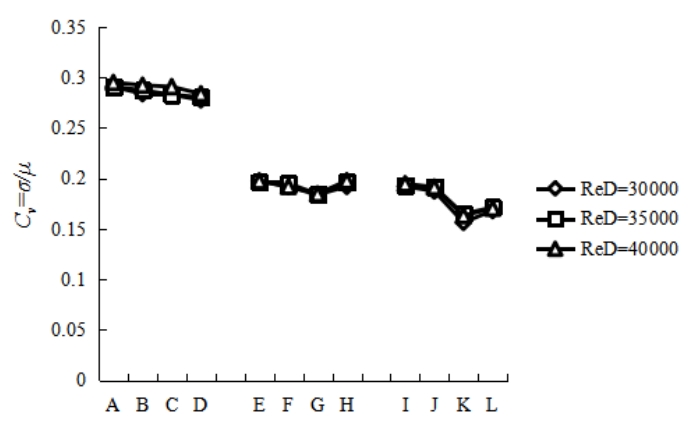

Fig. 15. Dispersion of $h$ under the same jet Reynolds number

For further studying the distribution of heat transfer on the film, we selected $h$ when $\operatorname{Re}_{D}=35000$ (as shown in Figure 16 to make an analysis. It can be seen from the figure that when the number of spanwise rows is 3,4 and 5 respectively, the corresponding D, G, K configurations have better uniform distributions of $h$. As the number of spanwise rows increases, the coverage area of hot air on the film and the heat transfer uniformity increase; the flow spacing $S_{l}$ determines the "wavelength" between the peaks of the $h$, which has a significant effect on the uniformity in the flow direction and is a key factor that affects the uniformity of the thickness of the film during stretching. From the could map of A-L shown in Figure 16, the maximum value of the surface heat transfer coefficient appears at a position far away from the inlet because of the large crossflow velocity $v_{c}$ in the crossflow section of the plenum chamber, which greatly affects the deflection of the jet velocity $v_{j}$. The angle $\beta$ between the synthetic velocity of the actual flow and the axis of the orifice satisfies:

$$
\tan \beta=\frac{v_{j}}{v_{c}}=\sqrt{\frac{P_{j}}{P_{c}}} .
$$

Besides, with the development of crossflow in the plenum chamber, the continuous decrease of flow rate reduces the crossflow pressure $P_{c}$; the increase of the static pressure $P_{j}$ leads to the increase of the jet velocity, which enhances the local heat transfer effect, but reduces the overall heat transfer uniformity as shown in Figure 17.
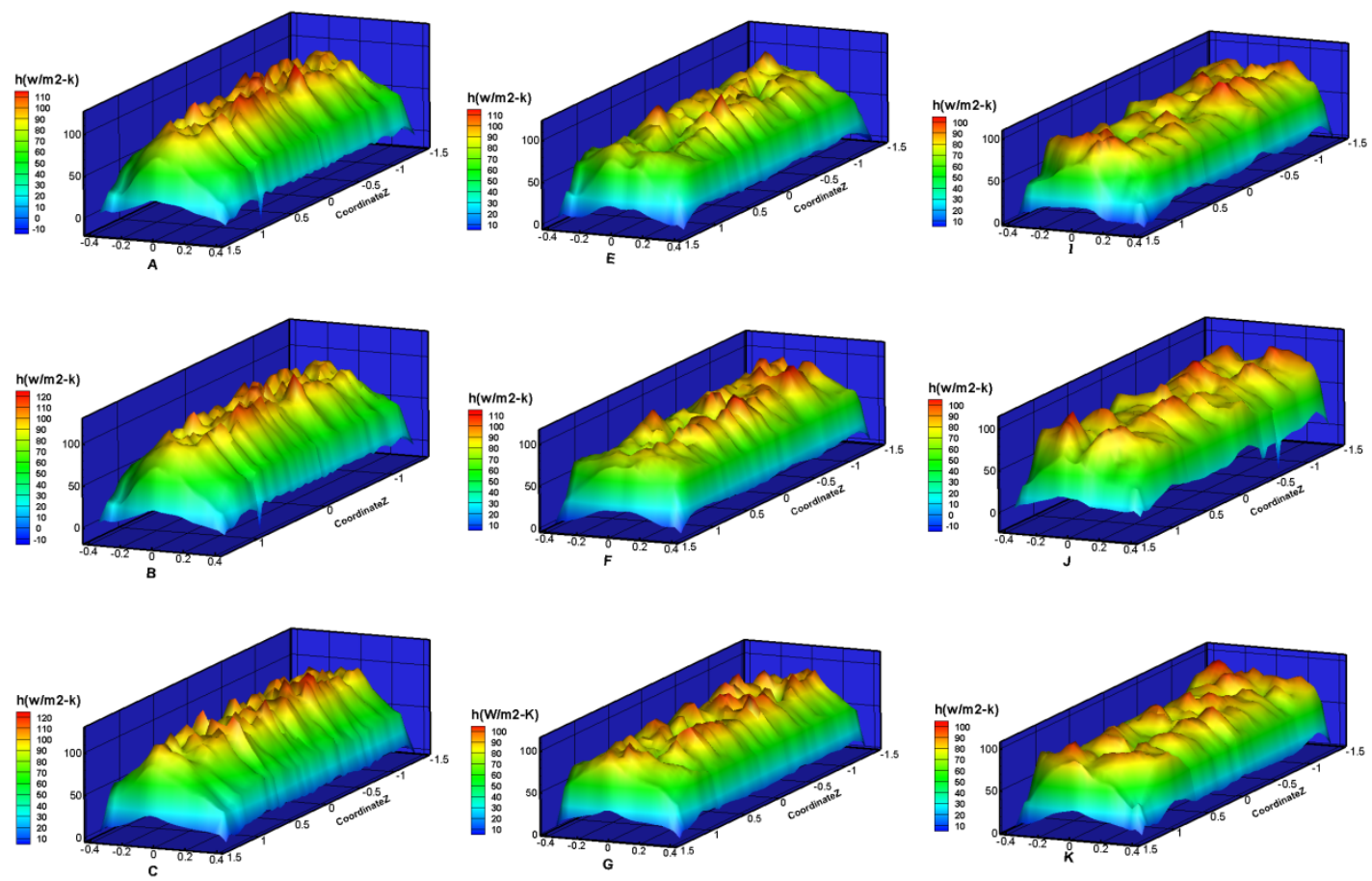


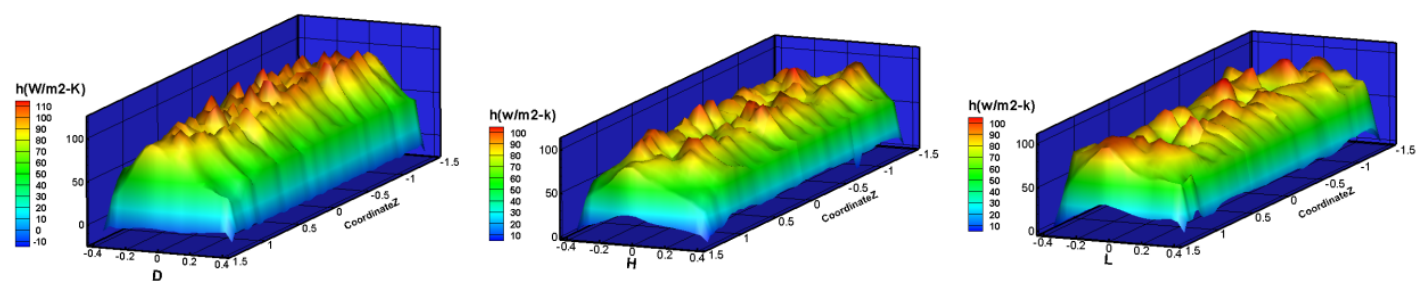

Fig.16. Distribution of the surface heat transfer coefficient h when $\operatorname{ReD}=35000$

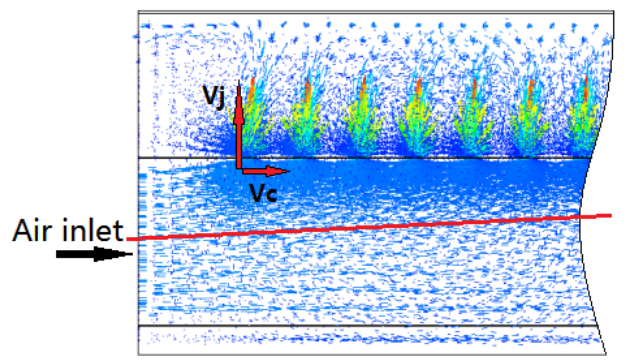

Fig. 17. Velocity vector of crossflow near the entrance

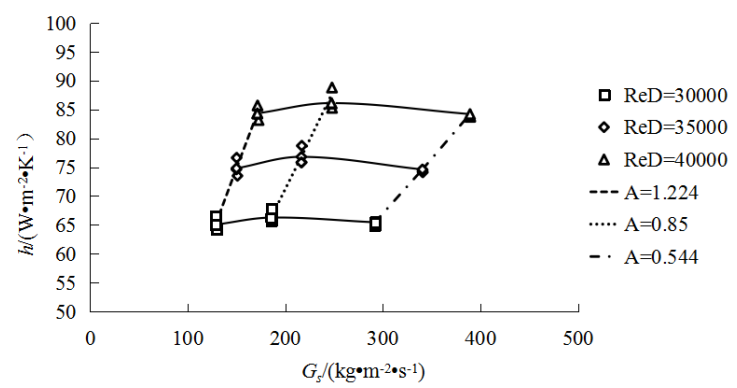

Fig. 18. Surface heat transfer coefficient $h$ of film varying with $G_{s}$

\subsection{Heat transfer efficiency}

Under the same velocity inlet, the supplying air volume of each plenum chamber is the same, while under the same jet Reynolds number, the supplying air volume of each plenum chamber with different orifice plate configurations needs to be adjusted according to the number of orifices in order to ensure that the jet Reynolds number of each orifice maintains consistent. In the production line, it is uneconomical to achieve good heat transfer effects with high volume of air supply, and the optimization goal of orifice plate is to reduce the amount of supplying air while increasing the surface heat transfer coefficient and improving the heat transfer uniformity. For the same heat transfer coverage area, i.e. the film surface area of $800 \times 2780 \mathrm{~mm}^{2}$, the mass flow rate per unit heat transfer area $G_{s}\left(\mathrm{~kg} / \mathrm{m}^{2} \cdot \mathrm{s}\right)$ corresponding to different configurations can reflect the hot air consumption through each orifice plate. Therefore, we can analyze $h$ varying with the mass flow rate per unit heat transfer area $G_{s}$ in each orifice plate configuration to estimate the heat transfer efficiency. Figure 18 shows $h$ of each configuration varies with $G_{s}$ under the same jet Reynolds number, where $G_{s}$ satisfies

$$
G_{s}=\frac{\pi \rho v_{j}}{A} .
$$

Where, $v_{j}$ is the jet velocity of orifice $(\mathrm{m} / \mathrm{s})$, and $A$ is the dimensionless area corresponding to a single orifice, $A=S_{1} \times S_{2}$ herein. From the above analysis, we can see that $h$ increases with the increase of jet Reynolds number, but the continuous increasing jet orifice Reynolds number may lead to the problem of crack or falling off from the clamp in the stretching process of the film. As can be seen from Figure 18, under the same jet Reynolds number, $h$ first increases and then decreases with the increase of $G_{s}$, and reaches the maximum value when $A=0.85$, namely with the number of spanwise rows as 4 . At this point, the number of orifices in the orifice plate is 100 , and the opening ratio

$$
A_{o}=\frac{\pi}{4} \frac{1}{\left(S_{1} / D\right)\left(S_{2} / D\right)}
$$

is 0.0208 .

\section{Summary}

1) Under the same boundary conditions, $h$ first increases and then decreases with the increase of staggered angle when the number of spanwise rows is 3 or 4 , and reaches the maximum at $30^{\circ} ; h$ is less affected by the staggered angle when the number of spanwise rows is 5 ; when the numbers of spanwise hole rows are respectively 3,4 , and 5, the minimum dispersion degree $C_{v}$ of $h$ occurred at 
the corresponding staggered angle of $45^{\circ}, 30^{\circ}, 30^{\circ}$, and then corresponding orifice plate configurations of $\mathrm{D}, \mathrm{G}, \mathrm{K}$ perform better in the uniformity of heat transfer.

2) A large velocity of initial crossflow in the plenum chamber will lead to an increase of the jet angle $\beta$; an increase of the static pressure at the end of the plenum chamber with long-channel enhances the local heat transfer effect but reduces the overall uniformity of heat transfer .

3) Under the same jet Reynolds number, $h$ first increases and then decreases with the increase of $G_{s}$, which indicates that increasing the opening ratio of orifice plate while increasing the amount of supplying hot air can not enhance the heat transfer strength continually. For better selection we should focus on the configurations with the number of spanwise rows as 4 and opening ratio as 0.0208 .

4) The orifice plate configurations with the number of spanwise rows as 4 and staggered angle as $30^{\circ}$ have higher heat transfer efficiency and a more uniform distribution, which can be used as a basis for local optimization further.

\section{References}

[1] Y. Hua, X. L. Gao, and X. F. Yue, Simulation and structural optimization of biaxial stretching temperature field distribution of double pull film equipment based on FLUENT, Mechanical Engineer, vol.3, pp. 81-83, 2014.

[2] L. Z. Xia, Q. Liu, and J. D. Shao, Numerical simulation and experimental study on flow fields of plenum chamber, Journal of Chemical Engineering of Chinese Universities, vol.31, pp. 712-719, 2017.

[3] A. Mohammadkhah and N. Mostoufi, Effect of geometry of the plenum chamber on gas distribution in a fluidized bed, Industrial and Engineering Chemistry Research, pp. 7624-7630, 2009.

[4] P. Mirek, J. Jablonski, and W. Nowak, Numerical optimization of air flow in the plenum chamber of an industrial CFB boiler, Canadian Journal of Microbiology, vol.52, pp. 1006-1015, 2008.

[5] Sachin V. Jangam, Arun S. Mujumdar, and Bhaskar N. Thorat, Design of an efficient gas distribution system for a fluidized bed dryer, Drying Technology, vol.27, pp. 1217-1228, 2009.

[6] P. P. Yang, Z. Wen, and R. F. Dou, Simulation of arrays of nozzle jet impinging heat transfer and its structural optimization, Energy for Metallurgical Industry, vol.30, pp. 16-19, 2011.

[7] W. Li, X. Li, and J. Ren, Effect of Reynolds number, hole patterns and hole inclination on cooling performance of an impinging jet array: Part I convective heat transfer results and optimization, Journal of Turbomachinery, vol.139, pp. 041002-041002-11, 2016.

[8] M. Goodro, J. Park, and P. Ligrani, Effects of hole spacing on spatially-resolved jet array impingement heat transfer, International Journal of Heat \& Mass Transfer, vol.51, pp. 62436253,2008 .

[9] M. Goodro, P. Ligrani, and M. Fox, Mach number, Reynolds number, jet spacing variations: Full array of impinging jets, Journal of Thermophysics \& Heat Transfer, vol.24, pp. 133-144,2015.

[10]J. Lee, Z. Ren, and P. Ligrani, Cross-flow effects on impingement array heat transfer with varying jet-to-target plate distance and hole spacing, International Journal of Heat \& Mass Transfer, vol.75, pp.534-544, 2014.

[11]T. Kanai and G. A. Campbell, "Film Processing", J. W. Wang, X. Q. Sun, and X. Q. Zuo, Chemical Industry Press, Beijing, 2003.

[12]W. Q. Tao, “Numerical heat transfer”, Xi'an Jiaotong University Press, Xi'an, 1988.

[13]X. Feng and C. H. He, "Principles of chemical engineering”, Science Press, Beijing, 2001. 